\title{
A Novel Approach to Combat Acquired Multiple Resistance in Escherichia coli by using EDTA as Efflux Pump Inhibitor
}

Manu Chaudhary, Shailesh Kumar and Anurag Payasi*

Department of Cell Culture and Molecular Biology, Venus Medicine Research Centre, Baddi, H.P. India

\begin{abstract}
In the present investigation, twenty three clinical isolates of Escherichia coli were analyzed for the presence of AcrAB-TolC efflux pump using the respective primer. In order to explore the possibility of EDTA as efflux inhibitor, the effect of EDTA on 4,6-diamidino-2-phenylindole (DAPI) efflux activity was measured. Thereafter, the minimum inhibitory concentration (MIC) of each antibacterial agent (drug) included in the study was determined with ethylene diamine tetraacetic acid (EDTA) and without EDTA according to Clinical and Laboratory Standards Institute, 2009. Next, the effect of EDTA and drugs with half of MIC on mRNA expressions of AcrAB-Tolc efflux pump was measured through semi quantitative reverse transcription-polymerase chain reaction (RT-PCR). Our results revealed that 10 mM EDTA when combined with ceftriaxone plus sulbactam (CSE1034; ceftriaxone plus sulbactam plus 10 mM EDTA) and meropenem, the MIC values reduced 32 and 8 fold, respectively, whereas other comparative drugs demonstrated only two fold reduction in MIC values. When, DAPI efflux activity was assessed with different concentration of EDTA a concentration-dependent inhibition of DAPI efflux was observed with a significant inhibition at $10 \mathrm{mM}$. Further, increasing the concentration of EDTA, failed to produce any more inhibition. Similarly, $10 \mathrm{mM}$ EDTA decreased the expression of AcrA, AcrB and TolC mRNA by 2.3, 2.2 and 2.4 folds, respectively. A significant $(p<0.0001)$ reduction of mRNA levels of AcrAB-TolC in CSE1034 treated groups was observed compared to meropenem $(p<0.01)$. However ceftriaxone, amoxycillin plus clavulanate, piperacillin plus tazobactam, and cefoperazone plus sulbactam treated group exhibited a non-significant changes $(p>0.05)$ in the expression of AcrAB-TolC mRNA compared with control. From the above results, it can be concluded that CSE1034 strongly decreased the expression of efflux pump. Hence, it can be one of the best treat infections caused by the microorganisms over expressing AcrAB-TolC efflux pump.
\end{abstract}

Keywords: Efflux pump; CSE1034; Minimum inhibitory concentration; Gene expression

\section{Introduction}

Escherichia coli is one of the leading pathogen responsible for severe ICU infections and have evolved a variety of strategies to resist antibiotics. A single resistance mechanism may diminish susceptibility to several therapeutic drugs allowing the survival of bacteria in their niches [1,2]. Among the various resistance mechanisms [3,4], antibiotic removal from the bacterial cells by efflux pumps are most common [57]. There are a number of reports indicating antibiotic resistance because of efflux pump in bacteria is increasing significantly [8,9]. Therefore, antibiotic efflux pumps are thought of as attractive therapeutic targets, where their inhibition can restore antibiotic activity.

To date, four multidrug efflux pumps have been characterized in E. coli, AcrAB-TolC, AcrEF-TolC, EmrB and EmrD [10]. The AcrABTolC system is the most prevalent and constitutively expressed in E. coli and found to be responsible for the antibiotic resistance [11], and is a tripartite complex containing AcrA, a fusion protein; AcrB, a cytoplasmic membrane transporter protein; and TolC, an outer membrane channel. Over expression of this pump is often associated with extrusion of most of the $\beta$-lactams antibiotics, leading to decreased susceptibility of antibiotics [12]. In recent years, in order to improve the susceptibility of antibiotics against the infections caused by clinical isolates with over expressing efflux pump, the use of efflux pump inhibitors have been investigated [13-15]. However, all these inhibitors have not yet met the requirements for application in clinical practice due to toxicity, immunosuppression, stability and solubility concerns [16]. Therefore, there is an urgent need of an efflux pump inhibitor which is to be safe and can be used in clinical practice. Very recently, effect of different concentrations of EDTA on MexA-MexB-OprM efflux pump of Pseudomonas aeruginosa and subsequent changes in susceptibility and expression has been studied [17]. Senad and Musaddiq [18] also observed the synergistics effects of combinations of EDTA and ciprofloxacin, gentamycin and ampicillin on various isolates of species of Pseudomonas, Proteus, E. coli and Klebsiella.

EDTA has been established by our lab to be safe upto $40 \mathrm{mg} /$ $\mathrm{kg} /$ body weight administered intravenously to Swiss albino mice (communicated for publication). Additionally, EDTA has been used intravenously in combination with vitamins and minerals in the treatment of various diseases including atherosclerotic vascular disease and renal ischemia [19-22]. Chaudhary et al. [23] investigated the sub acute intravenous toxicity of ceftriaxone plus sulbactam plus EDTA in Swiss albino mice and Sprague dawely rats (both male and female) and found to be safe upto $150 \mathrm{mg} / \mathrm{kg} /$ body weight. Very recently, the use of EDTA in ceftriaxone plus sulbactam combination has been reported in clinical case report [24,25].

In view of the above data, the current study was planned to evaluate the role of EDTA alone and in combination with various drugs on

*Corresponding author: Anurag Payasi, Venus Medicine Research Centre, Hill Top Industrial Estate, Bhatoli Kalan, Baddi, H.P.-173205 India, Tel: 91-1795302005; Fax: 91-1795-302133; E-mail: ccmb@venusremedies.com

Received September 26, 2012; Accepted October 25, 2012; Published October 29, 2012

Citation: Chaudhary M, Kumar S, Payasi A (2012) A Novel Approach to Combat Acquired Multiple Resistance in Escherichia coli by using EDTA as Efflux Pump Inhibitor. J Microb Biochem Technol 4: 126-130. doi:10.4172/1948-5948.1000082

Copyright: (C) 2012 Chaudhary M, et al. This is an open-access article distributed under the terms of the Creative Commons Attribution License, which permits unrestricted use, distribution, and reproduction in any medium, provided the original author and source are credited 
AcrAB-Tol C efflux pump. As far as authors know, this is the first report on E. coli where effect of different drugs has been compared on AcrAB-Tol C efflux pump with and without EDTA. For this purpose, E. coli clinical isolates were characterized based on the over expressing AcrAB-TolC efflux pump. In order to explore the possibility of EDTA as efflux inhibitor, the effect of EDTA on 4,6-diamidino-2-phenylindole (DAPI) efflux activity was measured. Thereafter, MIC of antibacterial agents was determined with ethylene diamine tetraacetic acid (EDTA) and without EDTA. Next, the effect of EDTA and drugs with half of MIC on mRNA expressions of AcrAB-Tolc efflux pump was measured.

\section{Materials and Methods}

\section{Antimicrobial agents}

A novel antibiotic adjuvant entity (AAE), with ceftriaxone sodium plus 10 mMEDTA disodium plus sulbactam sodium herein after referred to as CSE1034 (Elores of Venus Remedies Limited, Chandigarh, India), Ceftriaxone (Rocephin, Hoffmann-Laoche Pharmaceutical Limited, Basel Switzerland), amoxicillin plus clavulanate (Augmentin, Glaxo Smith Kline, Pharmaceuticals Limited, Mumbai, India), piperacillin plus tazobactam (Zosyn; Wyeth Pharmaceuticals, India, Mumbai), meropenem (Meronem, Astrazeneca Pharma India Ltd., Banglore, India), cefoperazone plus sulbactam (Magnex, Pfizer Limited, Mumbai, India) were used in the study. EDTA disodium was procured from Akzo Nobel Functional Chemicals B.V. Stationsstraat $773811 \mathrm{MH}$, P.O. Box 247, 3800 AE Amerfoort, Netherlands. All the drugs were reconstituted according to instructions of manufacturers. Working solutions were prepared using MH broth (Mueller Hinton, Himedia, Mumbai, India) and serial two fold dilutions were made using CAMH (Cation-Adjusted Mueller-Hinton, Himedia, Bombay, India) broth in wells of 96-well plate.

\section{Studied organisms}

A total of twenty three clinical isolates of E. coli collected from patients with urinary tract infection (UTI), bacteremia and pneumonia were used in this study. All of the isolates were obtained from four different medical colleges of North India including Postgraduate Institute of Medical Science, Lucknow (U.P), India, Vijayanagara Institute of Medical Sciences, Bareilly (U.P), India, Aligarh Muslim University, Aligarh (U.P), India, and Government Medical College and Hospital, Chandigarh, India. These isolates were then examined for the presence of the AcrAB-TolC efflux pump using the respective primers. Calcium-free buffer was used in the expression studies.

\section{Efflux pump activity assay}

AcrAB-TolC efflux activity was measured according to the method described elsewhere [26]. After preparation of cell suspension, the cell suspensions were pre-incubated for $5 \mathrm{~min}$ at $37^{\circ} \mathrm{C}$ with different concentrations of EDTA ( 0 to $11 \mathrm{mM}$ ) prior to the addition of glucose $(20 \mathrm{mM})$. The fluorescence of DAPI was measured at excitation and emission wavelengths of 355 and $457 \mathrm{~nm}$ respectively, with a Hitachi 2000 fluorescence spectrophotometer. The efflux of DAPI is monitored by the detection of a decrease in the level of fluorescence with time.

\section{MICs concentration}

MIC was determined according to CLSI, 2009 [27]. E. coli MTCC 739 was used as a control. The MIC value represents the lowest dilution at which bacteria fail to grow.

\section{DNA isolation}

Five $\mathrm{ml}$ of each bacterial culture was centrifuged at $5000 \mathrm{rpm}$ for 5 min at $4^{\circ} \mathrm{C}$ and pellet was washed once in Tris-EDTA (TE) buffer (Tris$\mathrm{HCl} 1.0 \mathrm{M}, \mathrm{pH}$ 8.0; EDTA $0.5 \mathrm{M}, \mathrm{pH}$ 8.0). After addition of $300 \mu \mathrm{l}$ of TE buffer, $40 \mu \mathrm{l}$ of $10 \%$ SDS (sodium dodecyl sulfate), $3 \mu \mathrm{l}$ of $0.5 \mathrm{M}$ EDTA ( $\mathrm{pH} \mathrm{8.0)}$ and incubated for $5 \mathrm{~min}$ at $65^{\circ} \mathrm{C}$. After incubation, $750 \mu \mathrm{l}$ of isopropanol was added. This was followed by centrifugation at 14,000 $\mathrm{rpm}$ for $5 \mathrm{~min}$ at $15^{\circ} \mathrm{C}$. The resulting pellet was resuspended in $500 \mu \mathrm{l}$ of TE and $2 \mu \mathrm{l}$ of mRNAse $\mathrm{A}(10 \mathrm{mg} / \mathrm{ml})$ and incubated at $65^{\circ} \mathrm{C}$ for 30 min, then added $2 \mu \mathrm{l}$ of proteinase $\mathrm{K}(20 \mathrm{mg} / \mathrm{ml})$ and incubated at $37^{\circ} \mathrm{C}$ for $15 \mathrm{~min}$. Following incubation, $1 \mathrm{ml}$ of phenol:chloroform (1:1) was added. The upper phase was transferred to another tube and added equal amount of chloroform, shaken well and centrifuged at 14,000 $\mathrm{rpm}$ for $5 \mathrm{~min}$ at $15^{\circ} \mathrm{C}$. The supernatant was provided with $40 \mu \mathrm{l}$ of $5 \mathrm{M}$ Na-Acetate ( $\mathrm{pH}$ 5.2) and $1 \mathrm{ml}$ of ethanol and left at room temperature for $1 \mathrm{~h}$, centrifuged at $7000 \mathrm{rpm}$ for $5 \mathrm{~min}$ at $4^{\circ} \mathrm{C}$. The DNA pellet was washed with $70 \%$ ethanol and suspended in $50 \mu \mathrm{l}$ of TE buffer. DNA purity and concentration were assayed in a spectrophotometer (260/280)

\section{Screening for efflux gene}

All the clinical isolates of $E$. coli were examined for the presence of the AcrAB-TolC genes using the respective primers. All the primers were obtained from Sigma Aldrich Chemicals Pvt. Ltd., Bangalore, India. Primers used for acrA forward-5'-CTCTCAGGCAGCTTAGCCCTAA-3' and reverse-5'-TGCAGAGGTTCAGTTTTGACTGTT; for acrB forward-5'- GGTCGATTCCGTTCTCCGTTA-3' and reverse-5' CTACCTGGAAGTAAACGTCATTGGT-3'; for tolC forward-5-AAGCCGAAAAACGCAACCT and reverse-5' CAGAGTCGGTAAGTGACCATC-3'. For PCR (polymerase chain reaction) amplifications, about $200 \mathrm{pg}$ of DNA was added to $20 \mu \mathrm{l}$ mixture containing $0.5 \mathrm{mM}$ of dNTPs, $1.25 \mu \mathrm{M}$ of each primer and $3.0 \mu \mathrm{l}$ of Taq polymerase (Banglore Genei) in 1x PCR buffer. Amplification was performed in a Eppendorf thermocycler (Germany) with cycling parameters, consisting of 40 cycles with $95^{\circ} \mathrm{C}$ for $10 \mathrm{~min}$ initial denaturation, $94^{\circ} \mathrm{C}$ denaturation $(1 \mathrm{~min}), 53^{\circ} \mathrm{C}$ annealing $(1 \mathrm{~min}), 72^{\circ} \mathrm{C}$ extension $(2 \mathrm{~min})$ and final extension $72^{\circ} \mathrm{C}$ for $7 \mathrm{~min}$. E. coli MTCC 739 was used as a control with lacking of AcrAB-TolC efflux pump. The PCR products were analyzed on a $1.5 \%$ agarose gel containing ethidium bromide (EtBr).

\section{Total mRNA extraction}

Total mRNA was isolated with Trizol (Invitrogen, USA) and processed for DNAse I treatment to remove traces of genomic DNA. The amount of extracted mRNA was quantified by measuring the absorbance at $260 \mathrm{~nm}$. The purity of the mRNA was checked by measuring the ratio of the absorbance at 260 and $280 \mathrm{~nm}$, where a ratio ranging from 1.8-2.0 was taken to be pure. The absence of degradation of the mRNA was confirmed by mRNA electrophoresis on a $1.5 \%$ agarose gel containing ethidium bromide $(\mathrm{EtBr})$.

\section{Reverse transcription-PCR analysis}

First strand cDNA was generated from $2 \mu \mathrm{g}$ of mRNA using the reverse transcriptase kit (Invitrogen, USA) according to the manufacturer's protocol. RT-PCR was performed as described in the RT-PCR kit (Invitrogen, USA) in a total volume of $20 \mu$ containing 5 $\mu \mathrm{l}$ of cDNA and per-designed gene-specific primers of $\operatorname{acr} A, \operatorname{acr} B$ and tolC. The $23 \mathrm{~S}$ rRNA was used as an internal control. Primer for the 23S rRNA gene was forward (5'-GTGTCAGGTGGGCAGTTTG-3') and reverse (5'-CATTCTGAGGGAACCTTTGG-3'). The cycling parameters for the PCR was as mentioned above. The RT-PCR products 
Citation: Chaudhary M, Kumar S, Payasi A (2012) A Novel Approach to Combat Acquired Multiple Resistance in Escherichia coli by using EDTA as Efflux Pump Inhibitor. J Microb Biochem Technol 4: 126-130. doi:10.4172/1948-5948.1000082

were analyzed by $1.5 \%$ agarose gel containing ethidium bromide.

\section{Effect of EDTA on efflux pump}

To investigate the effect of EDTA disodium on mRNA of AcrA, AcrB and TolC expression, three clinical isolates of $E$. coli positive for AcrAB-Tolc efflux pump was treated with various concentrations of EDTA. The same isolates without EDTA serve as a control.

\section{Effect of different drugs on efflux pump}

To assess the effect of drugs on mRNA expression of AcrA, AcrB and TolC, three clinical isolates of E. coli positive for AcrAB-Tolc efflux pump, were treated with drugs at half of MIC for 24 hours. The concentration of drugs used in this study is shown in Table 1. Following treatment, total mRNA was isolated with Trizol (Invitrogen, USA). First strand of cDNA was then synthesized from $2 \mu \mathrm{g}$ total mRNA using reverse transcriptase kit (Invitrogen, USA). The isolates without treatment with drug and EDTA serve as a control.

\section{Statistical analysis}

All data represent the mean \pm SD of three independent experiments. Data were analyzed using Graph pad prism 5.01 expressed as mean \pm

Table 1: Concentration of drugs used for the expression study.

\begin{tabular}{|c|c|c|c|c|c|}
\hline \multicolumn{5}{|c|}{ Concentration $(\mu \mathrm{g} / \mathrm{ml})$} \\
\hline Ceftriaxone & CSE1034 & $\begin{array}{c}\text { Amoxycillin+ } \\
\text { clavulanic acid }\end{array}$ & $\begin{array}{l}\text { Piperacillin+ } \\
\text { tazobactam }\end{array}$ & Meropenem & $\begin{array}{c}\text { Cefoperazone- } \\
\text { sulbactam }\end{array}$ \\
\hline 128 & 8 & 128 & 64 & 16 & 64 \\
\hline
\end{tabular}

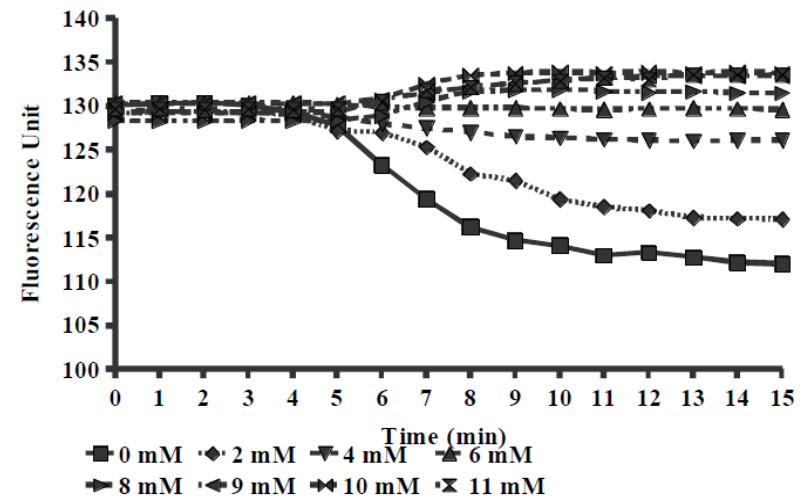

Figure 1: The levels of accumulation of 4,6-diamidino-2-phenylindole in three planktonic cultures of AcrAB-tolC positive clinical isolates of $E$. coli in the absence $(0 \mathrm{mM})$ and presence of various concentration of EDTA $(2,4,6,8$, $9,10$ and $11 \mathrm{mM})$. Results are presented in mean \pm SD of three independent experiments. standard deviation (SD). The continuous variables were tested with one-way analysis of variance (ANOVA) and dun-net test. Values of $\mathrm{P}>0.05$ were considered not to be significant.

\section{Results}

\section{Strain characterization for efflux pump}

Among the clinical isolates, only fifteen clinical isolates were found to be positive for AcrAB-TolC efflux pump and were included in the study.

\section{Effect of EDTA on efflux pump activity}

The activity of AcrAB-TolC efflux pump was monitored fluorometrically. Results of this study showed that in the absence of EDTA, the addition of glucose after 5 min resulted in a significant decrease of fluorescence as shown in Figure 1. When the reaction system was provided with different concentration of EDTA varying from 2 to $11 \mathrm{mM}$, a concentration-dependent inhibition of DAPI efflux was observed with a significant inhibition at $10 \mathrm{mM}$. Further increasing the concentration of EDTA, failed to produce any more inhibition.

\section{MIC}

MICs recorded for all of the antibacterial agents with and without EDTA against efflux pump positive clinical isolates and control (efflux negative) are summarized in Table 2. All the antibacterial agents without EDTA exhibited high MICs. For drugs, ceftriaxone, amoxycillin plus clavulanic acid, piperacillin plus tazobactam and cefeperazone plus sulbactam, there was only 2 fold reduction in MIC after addition of $10 \mathrm{mM}$ EDTA. Interestingly, when $10 \mathrm{mM}$ EDTA was added with ceftriaxone plus sulbactam (CSE1034; ceftriaxone plus sulbactam plus $10 \mathrm{mM}$ EDTA), 32 fold reduction in MIC was noted, whereas meropenem with $10 \mathrm{mM}$ EDTA demonstrated only 8 fold in MIC values of efflux positive clinical isolates. However, addition of $10 \mathrm{mM}$ EDTA in control group (efflux negative) did not demonstrate alteration in MIC.

\section{Effect of EDTA on expression of efflux pump}

Our results revealed that transporters (AcrA and AcrB) as well as outer membrane protein (Tol C) had higher expression in control groups, efflux positive which were not treated with EDTA/ drugs. However, EDTA was found to decrease the expression of the transporters mRNA (AcrA and AcrB) as well as outer membrane protein mRNA (Tol C) in comparison to control group. The downregulation of the expression of the AcrAB-TolC mRNA following treatment with EDTA occurred in a dose dependent manner with 2.3, 2.2 and 2.4 fold reduction, respectively, at $10 \mathrm{mM}$. Further, increasing the concentration of EDTA, no significant changes were noted (Table 3).

Table 2: Minimum inhibitory concentrations (MICs) of drugs on planktonic cultures of AcrAB-tolC positive clinical isolates and E. coli MTCC 739.

\begin{tabular}{|c|c|c|c|c|c|c|c|}
\hline & \multicolumn{7}{|c|}{$\mathrm{MIC}(\mu \mathrm{g} / \mathrm{ml})$} \\
\hline & 10 mM EDTA & $\mathrm{C}$ & $\mathrm{C}+\mathrm{S}$ & $A+C$ & $P+Z$ & $M$ & Cef $+S$ \\
\hline \multirow{2}{*}{ Efflux positive* $(n=15)$} & Without EDTA & $>512$ & $256-512$ & $>512$ & $256-512$ & $256-512$ & $256-512$ \\
\hline & With EDTA & 256 & $8-16$ & 256 & $128-256$ & $32-64$ & $128-256$ \\
\hline \multirow{2}{*}{$\begin{array}{c}\text { E.coli MTCC } 739 \\
\text { (efflux negative) }\end{array}$} & Without EDTA & 2 & 0.5 & 4 & 2 & 0.5 & 4 \\
\hline & With EDTA & 2 & 0.5 & 4 & 2 & 0.5 & 4 \\
\hline
\end{tabular}

C: Ceftriaxone; C+S: Ceftriaxone+sulbactam; P+Z: Piperacillin+tazobactam; A+C: Amoxicillin+clavulanic acid; cef+S: Cefoparazone+Sulbactam; M: Meropenem. *Efflux positive represents the presence of AcrAB-TolC overexpressing clinical isolates. 


\section{Effect of drugs on expression of efflux pump}

We also determined whether the expression of these genes was modified by following treatment with drugs. We observed that there was no significant change in mRNA levels of AcrAB-TolC in ceftriaxone, piperacillin+tazobactam, amoxycillin+clavulanate and cefoperazone+sulbactam treated group compared with control $(\mathrm{p}>0.05)$, whereas a significant $(\mathrm{p}<0.0001)$ decrease in mRNA levels of AcrAB-TolC in CSE1034 treated groups compared to meropenem treated groups $(\mathrm{p}<0.01)$ were noted (Figures $2 \mathrm{~A}, 2 \mathrm{~B}$ and $2 \mathrm{C}$ ).

Table 3: Effect of EDTA on expression of transporters and outer membrane protein in efflux pump of E.coli.

\begin{tabular}{|c|c|c|c|}
\hline $\begin{array}{c}\text { Concentration of EDTA } \\
(\mathrm{mM})\end{array}$ & AcrA mRNA & AcrB mRNA & TolC mRNA \\
\hline 0 & $88.54 \pm 7.4$ & $86.34 \pm 8.2$ & $87.52 \pm 8.6$ \\
\hline 2 & $82.34 \pm 8.1$ & $81.72 \pm 7.9$ & $82.78 \pm 8.2$ \\
\hline 4 & $70.32 \pm 6.5$ & $72.23 \pm 7.1$ & $69.32 \pm 6.8$ \\
\hline 8 & $55.53 \pm 4.9$ & $56.32 \pm 5.3$ & $54.94 \pm 5.1$ \\
\hline 10 & $38.34 \pm 2.8$ & $37.73 \pm 3.1$ & $36.23 \pm 2.4$ \\
\hline 12 & $35.21 \pm 3.5$ & $35.84 \pm 3.4$ & $34.86 \pm 3.8$ \\
\hline
\end{tabular}

Expression was determined by semi-quantitative RT-PCR, normalized to $23 \mathrm{~s}$ rRNA. Results are presented in mean \pm SD; 0=without EDTA.

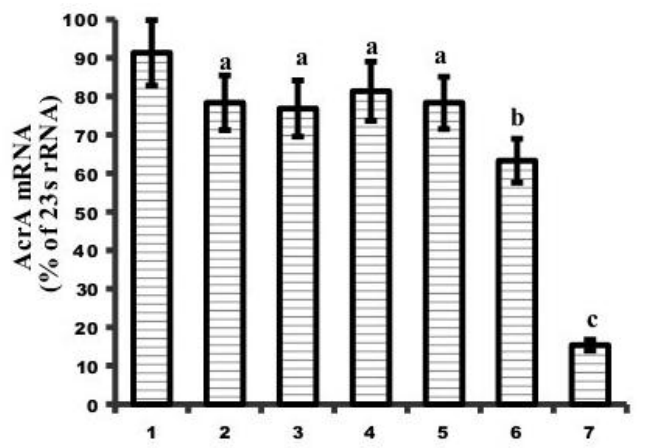

Figure 2a: AcrA expression was determined by semi-quantitative RT-PCR normalized to 23 s rRNA. $n=3$. mean \pm SD. ${ }^{a} p>0.05$ vs control; ${ }^{b} p<0.01$ vs control; ${ }^{c} p<0.0001$ vs control.

1. Control (without drug treatment); 2. Ceftriaxone; 3. Ceftriaxone+sulbactam; 4. Amoxicillin+clavulanic acid 5. Piperacillin+tazobactam; 6. Meropenem; 7. CSE1034.

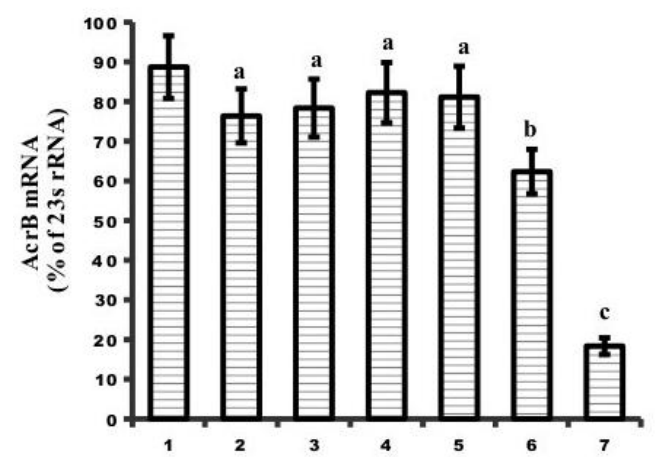

Figure 2b: AcrB expression was determined by semi-quantitative RT-PCR normalized to 23 s rRNA. $n=3$. mean $\pm S D$. ${ }^{a} p>0.05$ vs control; ${ }^{b} p<0.01$ vs control; ${ }^{c} p<0.0001$ vs control.

1. Control (without drug treatment); 2. Ceftriaxone; 3. Ceftriaxone+sulbactam; 4. Amoxicillin+clavulanic acid 5. Piperacillin+tazobactam; 6. Meropenem; 7. CSE1034.

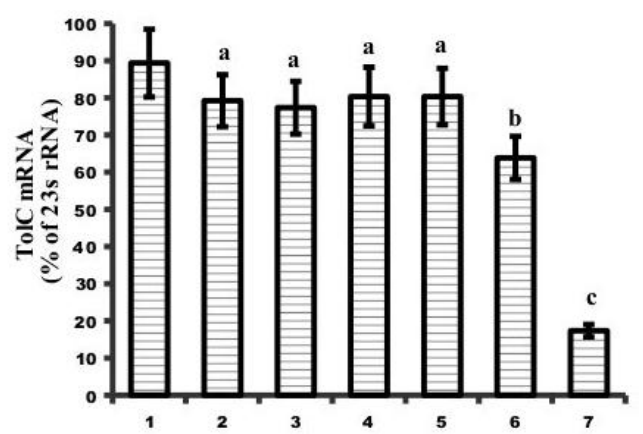

Figure 2c: TolC expression was determined by semi-quantitative RT-PCR normalized to $23 \mathrm{~s}$ rRNA. $n=3$. Mean \pm SD. ${ }^{a} p>0.05$ vs control; ${ }^{b} p<0.01$ vs control; ${ }^{c} p<0.0001$ vs control.

1. Control (without drug treatment); 2. Ceftriaxone; 3. Ceftriaxone+sulbactam; 4. Amoxicillin+clavulanic acid 5. Piperacillin+tazobactam; 6. Meropenem; 7 . CSE1034

\section{Discussion}

The efflux pumps encoded by bacteria confer clinically relevant resistance to antibiotics amongst other resistance mechanisms adopted by bacteria. Efflux pumps have a physiological role(s) and majority of gram-negative clinical isolates exhibits a multi drug-resistant phenotype because of over expressed efflux pumps [1].

Results of the present study indicated that when 10 mM EDTA was added into control group (efflux negative), there was no pronounced effect on MIC. However, when $10 \mathrm{mM}$ EDTA was combined with drugs, it reduced the MICs of efflux pump over expressing strains suggesting that it inhibits efflux pump. The maximum reduction in MIC (approximately 32 fold) was noted with CSE1034, probably, it caused strong inhibition of the efflux pump as a result increased accumulation of drug within the bacterial cells. Another probable reason of decreased MIC with CSE1034 could be synergistic effect of ceftriaxone plus sulbactam plus $10 \mathrm{mM}$ EDTA. It is worthy to mention here that our results of sub-acute intravenous toxicity of EDTA in mice when co-related with human dose revealed that EDTA upto $3.33 \mathrm{mg} /$ $\mathrm{kg}$ body weight is safe to human when administered intravenously. Interestingly, the $10 \mathrm{mM}$ concentration used in this study is within this range. On the other hand, the synergistic effect could not be found with other drugs hence there was less reduction in MIC, which might be because of failure of other drugs to respond AcrAB-TolC efflux pump synergistically. It has earlier been reported that decreased antibiotic accumulation via enhanced expression of drug efflux pumps is the major reason for resistance in gram-negative bacteria [7].

Our data revealed that the addition of EDTA into reaction system induced the inhibition of efflux pump activity with maximum inhibition at $10 \mathrm{mM}$ concentration. This is in accordance with the study reported for E. faecalis [28]. When various concentrations of EDTA was tested on efflux pump expression, it was found that $10 \mathrm{mM}$ EDTA decreased 43.33, 43.69 and $41.39 \%$ mRNA expression of AcrA, AcrB and TolC. The down-regulation of AcrA, AcrB and TolC following treatment with EDTA is probably due to chelation of calcium ions which results in disturbance in ATP production. It has earlier been reported that ATPases that hydrolyse ATP and provide protons for the activation of ABC-type transporters [29] are calcium dependent. Calcium is important for signaling and activating genetic systems [28], as well as for a wide variety of metabolic and energy-deriving pathways within the 
Citation: Chaudhary M, Kumar S, Payasi A (2012) A Novel Approach to Combat Acquired Multiple Resistance in Escherichia coli by using EDTA as Efflux Pump Inhibitor. J Microb Biochem Technol 4: 126-130. doi:10.4172/1948-5948.1000082

cell. It has been reported that the ATP concentration in E. coli directly controls the rate of mRNA transcription initiation [30], and therefore growth rate. Moreover, when drugs at half of MIC were studied, mRNA expression of AcrAB-Tolc were found to be decreased significantly with CSE1034, suggesting that increased antibiotic accumulation within the bacteria was associated with reduced expression of AcrAB-TolC mRNA. Similarly, it has also been suggested that artesunate enhance antibiotic susceptibility of $\beta$-lactams which is associated with reduced expression of AcrAB-TolC mRNA [31].

In conclusion, the over expression of the AcrAB-TolC pump in E. coli is responsible for decreased susceptibility of antibiotics. Our results implied that EDTA disodium enhances the susceptibility of $E$. coli efflux over expressed strains by inhibiting efflux pump which in turn increased accumulation of drugs inside the bacteria. The CSE1034 comprising ceftriaxone plus EDTA plus sulbactam showed significant reduction in efflux pump expression and hence can be considered as a choice of drug in E. coli infections with efflux over expressed strains.

\section{Acknowledgement}

Authors are thankful to the sponsor, Venus Pharma $\mathrm{GmbH}$, AM Bahnhof 1-3, D-59368, Werne, Germany, for providing assistance to carry out this study.

\section{References}

1. Piddock LJ (2006) Multidrug-resistance efflux pumps-not just for resistance. Nat Rev Microbiol 4: 629-636.

2. Poole K (2004) Efflux-mediated multiresistance in Gram-negative bacteria. Clin Microbiol Infect 10: 12-26.

3. Hawkey PM (1997) Resistance to carbapenems. J Med Microbiol 46: 451-454

4. Wright GD (1999) Aminoglycoside-modifying enzymes. Curr Opin Microbiol 2: 499-503.

5. Banerjee SK, Bhatt K, Misra P, Chakraborti PK (2000) Involvement of a natural transport system in the process of efflux-mediated drug resistance in Mycobacterium smegmatis. Mol Gen Genet 262: 949-956.

6. Marshall NJ, Piddock L (1997) Antibacterial efflux systems. Microbiologia 13 285-300.

7. Nikaido $H$, Zgurskaya $H I$ (2001) AcrAB and related multidrug efflux pumps of Escherichia coli. J Mol Microbiol Biotechnol 3: 215-218.

8. Levy SB (1992) Active efflux mechanisms for antimicrobial resistance. Antimicrob Agents Chemother 36: 695-703.

9. Webber MA, Piddock LJ (2003) The importance of efflux pumps in bacterial antibiotic resistance. J Antimicrob Chemother 51: 9-11.

10. Poole K (2000) Efflux-mediated resistance to fluoroquinolones in gram-negative bacteria. Antimicrob Agents Chemother 44: 2233-2241.

11. Zgurskaya HI, Nikaido H (2000) Multidrug resistance mechanisms: drug efflux across two membranes. Mol Microbiol 37: 219-225.

12. Yu EW, Aires JR, Nikaido H (2003) AcrB multidrug efflux pump of Escherichia coli: composite substrate-binding cavity of exceptional flexibility generates its extremely wide substrate specificity. J Bacteriol 185: 5657-5664.

13. Askoura M, Mottawea W, Abujamel T, Taher I (2011) Efflux pump inhibitors (EPIs) as new antimicrobial agents against Pseudomonas aeruginosa. Libyan J Med 6.

14. Lechner D, Gibbons S, Bucar F (2008) Plant phenolic compounds as ethidium bromide efflux inhibitors in Mycobacterium smegmatis. J Antimicrob Chemother 62: 345-348

15. Pages JM, Masi M, Barbe J (2005) Inhibitors of efflux pumps in Gram-negative bacteria. Trends Mol Med 11: 382-389.

16. Lomovskaya O, Warren MS, Lee A, Galazzo J, Fronko R, et al. (2001) Identification and characterization of inhibitors of multidrug resistance efflux pumps in Pseudomonas aeruginosa: novel agents for combination therapy. Antimicrob Agents Chemother 45: 105-116.

17. Chaudhary M, Payasi A (2012) Ethylenediaminetetraacetic acid: A non antibiotic adjuvant enhancing Pseudomonas aeruginosa susceptibility. Afr $J$ Microbiol Res 6: 6799-6804.

18. Senad KS, Musaddiq M (2011) In vitro efficacy testing of EDTA in combination with antibiotics against prominent pathogens. Biosci Biotech Res Comm 4: 41 46.

19. Ernst $E$ (2000) Chelation therapy for coronary heart disease: An overview of al clinical investigations. Am Heart J 140: 1139-141.

20. Foglieni C, Fulgenzi A, Ticozzi P, Pellegatta F, Sciorati C, et al. (2006) Protective effect of EDTA preadministration on renal ischemia. BMC Nephrol 7: 5.

21. Jackson JA, Riordon HD (1992) Improvement of essential hypertension after EDTA infusion treatment. $\mathrm{J}$ Orthomol Med 7: 1-16.

22. Lewin MR (1997) Chelation therapy for cardiovascular disease. Review and commentary. Tex Heart Inst J 24: 81-89.

23. Chaudhary M, Sudaroli M, Krishnaraju V (2011) Evaluation of sub acute toxicity profile of fixed dose combination of ceftriaxone plus sulbactam plus ethylenediaminetetraacetic acid in swiss albino mice and Sprague dawley rats. J Pharm Res 4: 4511-4514.

24. Ragunath MP, Mitra D, Sasmal D (2011) Case reports: otitis media treatment with sulbactomax. Pharmacologyonline 2: 8-11.

25. Sudaroli M, Ragunath MP, Sasmal D (2011) Treatment of septic arthritis with the combination of ceftriaxone plus sulbactum plus EDTA (sulbactomax) in the current scenario of resistance: a case report. Pharmacologyonline 3: 3-5.

26. Lee EW, Chen J, Huda MN, Kuroda T, Mizushima T, et al. (2003) Functiona cloning and expression of emeA, and characterization of EmeA, a multidrug efflux pump from Enterococcus faecalis. Biol Pharm Bull 26: 266-270.

27. Clinical and Laboratory Standards Institute (2009) Performance standards for antimicrobial susceptibility testing, $19^{\text {th }}$ informational supplement, CLSI document M100-S19, Wayne, PA.

28. Naseem R, Wann KT, Holland IB, Campbell AK (2009) ATP regulates calcium efflux and growth in E. coli. J Mol Biol 391: 42-56.

29. Martins A, Machado L, Costa S, Cerca P, Spengler G, et al. (2011) Role of calcium in the efflux system of Escherichia coli. Int $\mathrm{J}$ Antimicrob Agents 37: 410-414.

30. Schneider DA, Gourse RL (2004) Relationship between growth rate and ATP concentration in Escherichia coli: a bioassay for available cellular ATP. J Bio Chem 279: 8262-8268.

31. Li B, Yao Q, Pan XC, Wang N, Zhang R, et al. (2011) Artesunate enhances the antibacterial effect of \{beta\}-lactam antibiotics against Escherichia coli by increasing antibiotic accumulation via inhibition of the multidrug efflux pump system AcrAB-TolC. J Antimicrob Chemother 66: 769-777. 